\title{
Analytical Probe Compensation Under Transient Signals Using a Transfer Function Approach
}

\author{
Francinei L. Vieira ${ }^{(1)(2)}$ \\ (1) Leibniz University Hannover \\ (2) University of Nottingham \\ Hannover, Germany \\ vieira@geml.uni-hannover.de
}

\author{
Muhammad Septian Alamsyah (1) (2) \\ (1) Leibniz University Hannover \\ (2) University of Nottingham \\ Hannover, Germany \\ alamsyah@geml.uni-hannover.de
}

\begin{abstract}
A larger deployment of power-electronic converters, with increasingly high switching frequencies, can be observed and associated with a higher complexity to achieve optimal performance not only on design, integration, reliability, and cost of power solutions but also on related electromagnetic compatibility measurements. Thus, a pulsed sensor calibration over the $\mathrm{kHz}$ range is desired to obtain significant results. This work presents a mathematical derivation of a transient compensation technique for current clamps based on transfer functions and time-domain waveforms. By converting the aperiodic signals of the probe using a Laplace transform, the method can be easily performed in the frequency domain, the original waveform in the line can be accurately predicted and correction factors for field sensors can be determined if needed.
\end{abstract}

Keywords-compensation; EMC; double exponential pulse shape; transfer characteristic; transfer function.

\section{INTRODUCTION}

Transient analysis is gaining more relevance due to the increasing deployment of power electronics. Low and highvoltage modern applications, such as electrical vehicles, power supplies, wireless networks, and the internet of things inserted into the market usually need to attend to requirements within their respective standards of electromagnetic compatibility (EMC) [1].

The behaviour prediction of a system includes some degree of inaccuracy, since the theoretical or empirical formulation of a real system considers only features of greatest importance, and never take all the aspects into account. Instead of analysing complex systems at once, a better approach is to examine simpler subsystems and combine them when necessary [2]. In [3], uncertainty evaluation of transient phenomena was observed by characterizing the dynamic performance of both analogue and digital sections of an oscilloscope. Errors of transient current measurement using loop probes are covered in [4]. Measurements of transient field impulses were done using a spherical sensor and optical insulation system in [5] and then reconstructed using Wavelet transformation.

Upcoming silicon carbide ( $\mathrm{SiC}$ ) and gallium nitride ( $\mathrm{GaN})$ bandgap semiconductors give some flexibility on the switching frequency, ranging from a few $\mathrm{kHz}$ on high-power applications to several $\mathrm{MHz}$ for low-power procedures. Therefore, measurement techniques are inherent to characterize complex devices and their interconnections, for example, in conducted or radiated emission tests [6]. In this regard, a method to substitute EMC antenna measurements with field and cable scan with computed transfer functions are

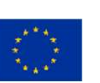

This project has received funding from the European Union's Horizon 2020 research and innovation programme under the Marie Skłodowska-Curie grant agreement No 812753. described in [7]. Consequently, the measuring apparatus must be regularly verified for compliance, i.e., if the error between the received variable and the real system variable is within the expected margin.

EMC involves not only hardware but also software. As many measurement devices obtain the time function and only then calculate the signal spectrum using, e.g., the Fast Fourier Transform (FFT), the input signal might be affected by the poor parameter selection or by the non-ideal receiving characteristic of a probe, which are the main sources of measurement errors [8]. Moreover, transient field sensor calibration is not suitable with the traditional frequencydomain calibration.

In this work, preliminary analyses involving time and frequency-domain with known waveforms and basic transfer functions are presented, as an introduction for the probe compensation technique. While a time-domain representation provides a quick insight into the dynamics of spurious signals, amplitude and phase information, a frequency-domain analysis can display the spectrum of a signal, which provides valuable information about the bandwidth and the composition of the most important signal frequencies, for example. The calculation between time and frequency domains can be related using the Fourier or Laplace analysis, focused on the $\mathrm{kHz}$ and $\mathrm{MHz}$ range.

\section{COMPENSATION THEORY}

The methodology includes the use of time and frequencydomain analyses with known waveforms and a simple representative circuit. The transfer characteristic of a low-pass filter represents the bandwidth limitation of a measuring chain. Its output is compared with the stimulus signal: a double-exponential pulse, which can represent an overshoot due to power-switching or a lightning effect.

Since the output signal differs from the input, the transfer characteristic can be used for compensation of the output signal easier in the frequency domain. By deriving the transfer function $H(s)$, e.g., of a current clamp, the real variable, $g(t)$ can be predicted from the measured variable $f(t)$, according to the Fig. 1.

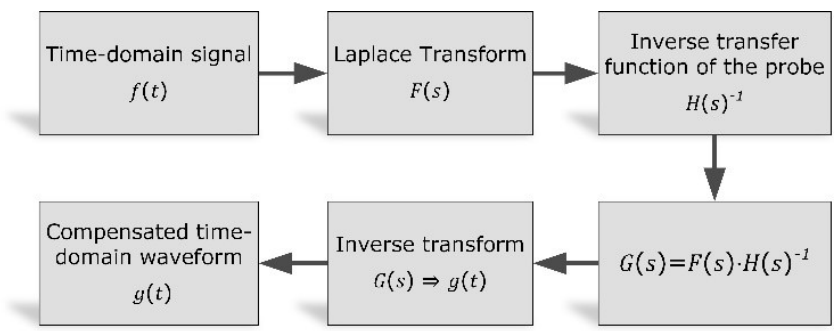

Fig. 1 - Block diagram of the signal compensation process 
A double-exponential pulse is used as the reference $g(t)$. This pulse was chosen because of its fast rise time and wideband frequency and is commonly associated with highpower electromagnetic (HEMP) threats to electronic systems [9]. Its output, $f(t)$, is the signal to be compensated and has the spectrum described in (1) and the resulting output time function indicated in (2). A low-pass filter is used as the allegedly transfer function $H(s)$ of the probe, just for demonstration of the method.

The time function convolution becomes a product of their transfer functions in the s-domain. Thus, the output spectrum of the signal to be compensated is described in (1) and (2).

$$
\begin{gathered}
F(s)=\underbrace{\left(\frac{1 / \tau}{s+1 / \tau}\right)}_{\text {Low-pass filter }} \cdot \underbrace{\frac{\beta-\alpha}{(s+\alpha)(s+\beta)}}_{\text {Double exponential pulse }} \\
F(s)=\frac{(\beta-\alpha) e^{-t / \tau}}{(\alpha \tau-1)(\beta \tau-1)\left(s+\frac{1}{\tau}\right)}+\frac{1}{(\alpha \tau-1)(s+\alpha)} \\
-\frac{1}{(\beta \tau-1)(s+\beta)}
\end{gathered}
$$

Where $\alpha$ and $\beta$ are parameters of the double-exponential pulse function representing a $1.2 \times 50 \mu \mathrm{s}$ waveform. $\tau$ is the time constant of the low-pass filter. The output time function is obtained after an inverse Laplace transform as shown in (3).

$$
g(t)=\frac{\tau(\beta-\alpha) e^{-t / \tau}}{(\alpha \tau-1)(\beta \tau-1)}+\frac{e^{-\alpha t}}{1-\alpha \tau}-\frac{e^{-\beta t}}{1-\beta \tau}
$$

This waveform is the signal to be compensated using the inverse transfer function (4) and is discussed in the next section.

$$
H^{-1}(s)=\left(\frac{s+1 / \tau}{1 / \tau}\right)
$$

\section{DISCUSSION AND RESULTS}

The time function of the double exponential pulse is shown in Fig. 2. The input pulse (blue) supplies the low-pass filter, which has a cut-off frequency of $5 \mathrm{kHz}$. As a result, a

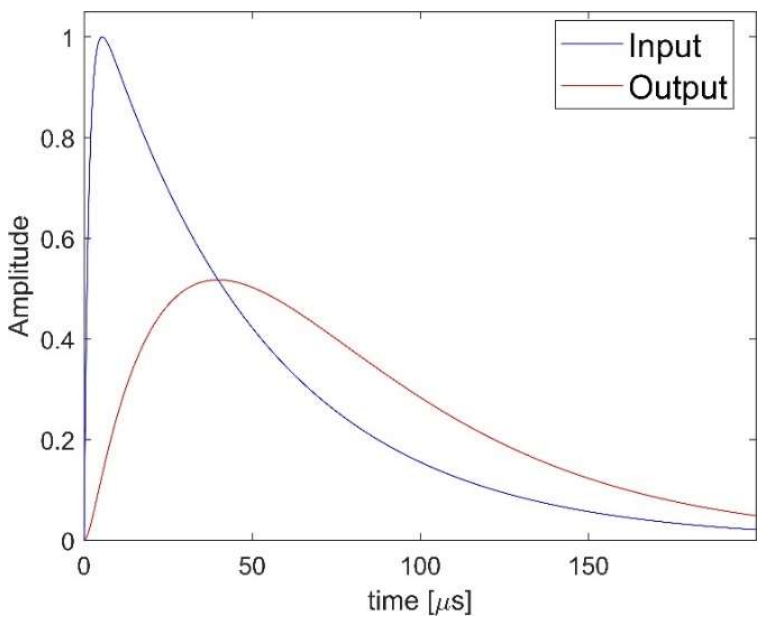

Fig. 2. Input and output in the time-domain damping effect is observed on the output (red), as seen in Fig. 3. The spectra of the input and output match on the flat region, but the low-pass filter behaviour attenuates the highfrequency components after the cut-off frequency.

The magnitude is damped by almost $50 \%$ and the rise time of the output waveform is 15 times bigger than the input. In real-life applications, such as for transient wave protection design, this output could lead to an erroneous specification.

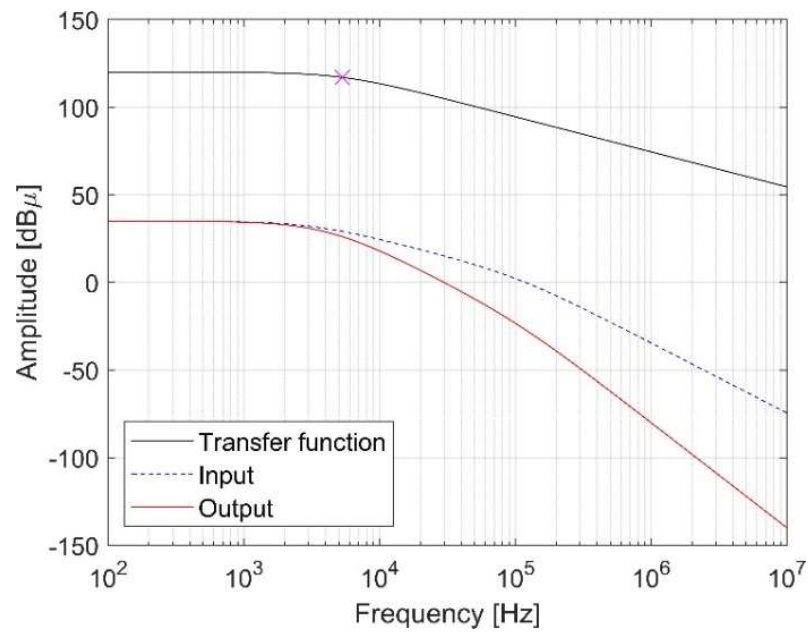

Fig. 3. Transfer function and spectra of the input and output signals (b).

The transfer characteristic of the current clamp is not constant over the frequency range, damping important components on a transient signal. This could be solved by applying the output signal to the inverse transfer function and the result is shown in Fig. 4 with agreeable precision.

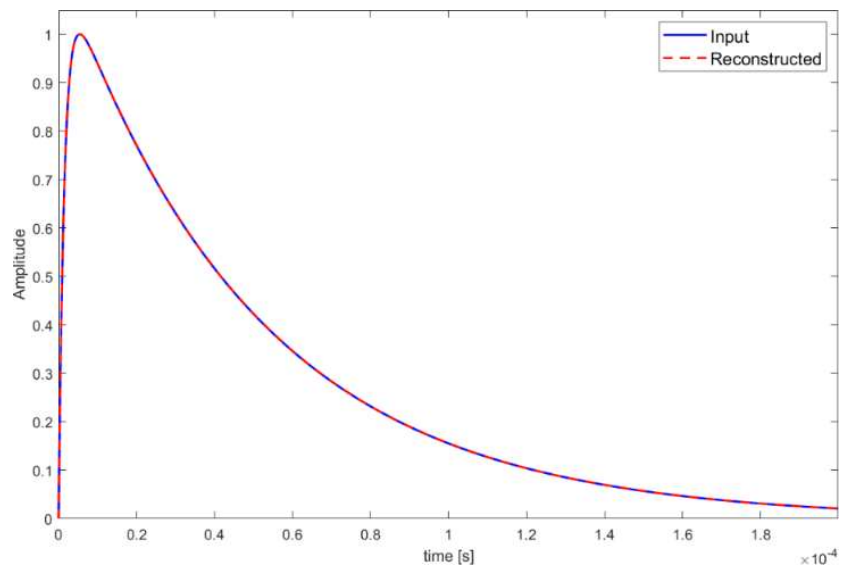

Fig. 4. Comparison between the reconstructed and ideal waveforms

And even considering different cut-off frequencies, the compensation method could lead to similar behaviours with good accuracy.

\section{CONCLUSIONS}

This methodology demonstrated a good agreement to predict a known double exponential pulse waveform using transfer functions. A potential limitation may occur due to truncated signals and background noise, which lead to some challenges in signal processing and analytic modelling. Those aspects are still to be confronted with measurements. 
The frequency-domain used here with Laplace transform is more adequate for non-periodic waveforms, but many digital measurement devices use an FFT for frequencydomain analysis, which is more suitable to periodic waveforms but it can be extended to non-periodic signals with additional considerations, like windowing and aliasing.

As real systems have transfer functions of higher complexity, a virtual network analyser (VNA) can be used for an accurate derivation of the current clamp's transfer characteristic between the observed magnetic field in the transmission lines and the voltage output of the probe, especially for relevant frequencies in the $\mathrm{kHz}$ range. This step is essential for the further development of this work.

\section{ACKNOWLEDGMENT}

The authors thank Prof. Heyno Garbe for the valuable suggestions.

\section{REFERENCES}

[1] M. Sigmund and L. Brancik, "Requirements on Needed Frequency Bandwidth Depending on Pulse Waveforms and Their Allowed Distortion," J. Electr. Eng., vol. 67, no. 6, pp. 459-462, Dec. 2016, doi: 10.1515/jee-2016-0068.

[2] K. S. Lee, Ed., EMP Interaction: Principles, Techniques and Reference Data, 1st ed. Albuquerque, USA: Air Force Weapons Laboratory, 1980.
[3] A. Moschitta, F. Stefani, and D. Petri, "Measurements of Transient Phenomena With Digital Oscilloscopes," IEEE Trans. Instrum. Meas., vol. 56, no. 6, pp. 2486-2491, Dec. 2007, doi: 10.1109/TIM.2007.908119.

[4] R. Cao, J. Li, Q. Jiao, and J. Yuan, "Analysis and measure the transient currents of rail-gun with loop probes," in 2012 16th International Symposium on Electromagnetic Launch Technology, May 2012, vol. 41, no. 5, pp. 1-5, doi: 10.1109/EML.2012.6325122.

[5] Qingquan Li, Yanming Li, Dake Xu, and Junling Cao, "Measurement of transient electric fields and analysis based on wavelet," in Proc. of Int. Symp. on Elec. Insulating Materials (ISEIM 2001). Asian Conference on Electrical Insulating Diagnosis (ACEID 2001). 33rd Symposium on Elec. and Electr. Insulating Materials and App. in System, 2001, no. 710049, pp. 233-236, doi: 10.1109/ISEIM.2001.973627.

[6] F. Leferink, "Conducted Interference, Challenges and Interference Cases," IEEE Electromagn. Compat. Mag., vol. 4, no. 1, pp. 78-85, 2015, doi: 10.1109/MEMC.2015.7098517.

[7] D. Rinas, J. Jia, A. Zeichner, and S. Frei, "Substituting EMC emission measurement by field and cable scan method using measured transfer function," Adv. Radio Sci., vol. 11, pp. 183-188, Jul. 2013, doi: 10.5194/ars-11-183-2013.

[8] A. Tankielun, H. Garbe, and J. Werner, "Calibration of electric probes for post-processing of near-field scanning data," in IEEE International Symposium on Electromagnetic Compatibility, 2006, vol. 1, no. 2, pp. 119-124, doi: 10.1109/ISEMC.2006.1706276.

[9] M. Camp and H. Garbe, "Parameter Estimation of Double Exponential Pulses (EMP, UWB) With Least Squares and Nelder Mead Algorithm," IEEE Trans. Electromagn. Compat., vol. 46, no. 4, pp. 675-678, Nov. 2004, doi: 10.1109/TEMC.2004.838228. 